CERN PPE/96-086

3 July 1996

\title{
Search for charginos and neutralinos with R-parity violation at $\sqrt{s}=130$ and $136 \mathrm{GeV}$
}

\author{
The ALEPH Collaboration*)
}

\begin{abstract}
Searches for charginos and neutralinos produced in $\mathrm{e}^{+} \mathrm{e}^{-}$collisions at centre-ofmass energies of 130 and $136 \mathrm{GeV}$ have been performed under the assumptions that $\mathrm{R}$-parity is not conserved, that the dominant R-parity violating coupling involves only leptonic fields, and that the lifetime of the lightest supersymmetric particle can be neglected. In the $5.7 \mathrm{pb}^{-1}$ data sample collected by ALEPH, no candidate events were found. As a result, chargino and neutralino masses and couplings are constrained and the domains previously excluded at LEP1 are extended.
\end{abstract}

(Submitted to Physics Letters B)

*) See next pages for the list of authors 


\section{The ALEPH Collaboration}

D. Buskulic, I. De Bonis, D. Decamp, P. Ghez, C. Goy, J.-P. Lees, A. Lucotte, M.-N. Minard, P. Odier, B. Pietrzyk

Laboratoire de Physique des Particules (LAPP), IN ${ }^{2} P^{3}-C N R S, 74019$ Annecy-le-Vieux Cedex, France

M.P. Casado, M. Chmeissani, J.M. Crespo, M. Delfino, ${ }^{12}$ I. Efthymiopoulos, ${ }^{20}$ E. Fernandez, M. Fernandez-Bosman, Ll. Garrido, ${ }^{15}$ A. Juste, M. Martinez, S. Orteu, A. Pacheco, C. Padilla, A. Pascual, J.A. Perlas, I. Riu, F. Sanchez, F. Teubert

Institut de Fisica d'Altes Energies, Universitat Autonoma de Barcelona, 08193 Bellaterra (Barcelona), Spain $^{7}$

A. Colaleo, D. Creanza, M. de Palma, G. Gelao, M. Girone, G. Iaselli, G. Maggi, ${ }^{3}$ M. Maggi, N. Marinelli, S. Nuzzo, A. Ranieri, G. Raso, F. Ruggieri, G. Selvaggi, L. Silvestris, P. Tempesta, G. Zito

Dipartimento di Fisica, INFN Sezione di Bari, 70126 Bari, Italy

X. Huang, J. Lin, Q. Ouyang, T. Wang, Y. Xie, R. Xu, S. Xue, J. Zhang, L. Zhang, W. Zhao

Institute of High-Energy Physics, Academia Sinica, Beijing, The People's Republic of China ${ }^{8}$

R. Alemany, A.O. Bazarko, M. Cattaneo, P. Comas, P. Coyle, H. Drevermann, R.W. Forty, M. Frank, R. Hagelberg, J. Harvey, P. Janot, B. Jost, E. Kneringer, J. Knobloch, I. Lehraus, E.B. Martin, P. Mato,

A. Minten, R. Miquel, Ll.M. Mir ${ }^{2}$ L. Moneta, T. Oest, ${ }^{1}$ F. Palla, J.-F. Pusztaszeri, F. Ranjard, P. Rensing, L. Rolandi, D. Schlatter, M. Schmelling, ${ }^{24}$ O. Schneider, W. Tejessy, I.R. Tomalin, A. Venturi, H. Wachsmuth, A. Wagner

European Laboratory for Particle Physics (CERN), 1211 Geneva 23, Switzerland

Z. Ajaltouni, A. Barrès, C. Boyer, A. Falvard, P. Gay, C . Guicheney, P. Henrard, J. Jousset, B. Michel, S. Monteil, J-C. Montret, D. Pallin, P. Perret, F. Podlyski, J. Proriol, J.-M. Rossignol

Laboratoire de Physique Corpusculaire, Université Blaise Pascal, IN ${ }^{2} P^{3}-C N R S$, Clermont-Ferrand, 63177 Aubière, France

T. Fearnley, J.B. Hansen, J.D. Hansen, J.R. Hansen, P.H. Hansen, B.S. Nilsson, A. Wäänänen

Niels Bohr Institute, 2100 Copenhagen, Denmark ${ }^{9}$

A. Kyriakis, C. Markou, E. Simopoulou, I. Siotis, A. Vayaki, K. Zachariadou

Nuclear Research Center Demokritos (NRCD), Athens, Greece

A. Blondel, J.C. Brient, A. Rougé, M. Rumpf, A. Valassi, ${ }^{6}$ H. Videau ${ }^{21}$

Laboratoire de Physique Nucléaire et des Hautes Energies, Ecole Polytechnique, IN ${ }^{2} P^{3}-C N R S, 91128$ Palaiseau Cedex, France

E. Focardi, ${ }^{21}$ G. Parrini

Dipartimento di Fisica, Università di Firenze, INFN Sezione di Firenze, 50125 Firenze, Italy

M. Corden, C. Georgiopoulos, D.E. Jaffe

Supercomputer Computations Research Institute, Florida State University, Tallahassee, FL 323064052, USA $^{13,14}$

A. Antonelli, G. Bencivenni, G. Bologna, ${ }^{4}$ F. Bossi, P. Campana, G. Capon, D. Casper, V. Chiarella, G. Felici, P. Laurelli, G. Mannocchi, ${ }^{5}$ F. Murtas, G.P. Murtas, L. Passalacqua, M. Pepe-Altarelli

Laboratori Nazionali dell'INFN (LNF-INFN), 00044 Frascati, Italy

L. Curtis, S.J. Dorris, A.W. Halley, I.G. Knowles, J.G. Lynch, V. O'Shea, C. Raine, P. Reeves, J.M. Scarr, K. Smith, A.S. Thompson, F. Thomson, S. Thorn, R.M. Turnbull

Department of Physics and Astronomy, University of Glasgow, Glasgow G12 8QQ,United Kingdom ${ }^{10}$ 
U. Becker, C. Geweniger, G. Graefe, P. Hanke, G. Hansper, V. Hepp, E.E. Kluge, A. Putzer, B. Rensch, M. Schmidt, J. Sommer, H. Stenzel, K. Tittel, S. Werner, M. Wunsch

Institut für Hochenergiephysik, Universität Heidelberg, 69120 Heidelberg, Fed. Rep. of Germany ${ }^{16}$

D. Abbaneo, R. Beuselinck, D.M. Binnie, W. Cameron, P..J. Dornan, A. Moutoussi, J. Nash, J.K. Sedgbeer, A.M. Stacey, M.D. Williams

Department of Physics, Imperial College, London SW7 2BZ, United Kingdom ${ }^{10}$

G. Dissertori, P. Girtler, D. Kuhn, G. Rudolph

Institut für Experimentalphysik, Universität Innsbruck, 6020 Innsbruck, Austria ${ }^{18}$

A.P. Betteridge, C.K. Bowdery, P. Colrain, G. Crawford, A.J. Finch, F. Foster, G. Hughes, T. Sloan, M.I. Williams

Department of Physics, University of Lancaster, Lancaster LA1 4YB, United Kingdom ${ }^{10}$

A. Galla, A.M. Greene, C. Hoffmann, K. Kleinknecht, G. Quast, B. Renk, E. Rohne, H.-G. Sander, P. van Gemmeren C. Zeitnitz

Institut für Physik, Universität Mainz, 55099 Mainz, Fed. Rep. of Germany ${ }^{16}$

J.J. Aubert, ${ }^{21}$ A.M. Bencheikh, C. Benchouk, A. Bonissent, ${ }^{21}$ G. Bujosa, D. Calvet, J. Carr, C. Diaconu, N. Konstantinidis, P. Payre, D. Rousseau, M. Talby, A. Sadouki, M. Thulasidas, A. Tilquin, K. Trabelsi

Centre de Physique des Particules, Faculté des Sciences de Luminy, IN ${ }^{2} P^{3}-$ CNRS, 13288 Marseille, France

M. Aleppo, F. Ragusa ${ }^{21}$

Dipartimento di Fisica, Università di Milano e INFN Sezione di Milano, 20133 Milano, Italy

I. Abt, R. Assmann, C. Bauer, W. Blum, H. Dietl, F. Dydak, ${ }^{21}$ G. Ganis, C. Gotzhein, K. Jakobs, H. Kroha, G. Lütjens, G. Lutz, W. Männer, H.-G. Moser, R. Richter, A. Rosado-Schlosser, S. Schael, R. Settles, H. Seywerd, R. St. Denis, W. Wiedenmann, G. Wolf

Max-Planck-Institut für Physik, Werner-Heisenberg-Institut, 80805 München, Fed. Rep. of Germany ${ }^{16}$

J. Boucrot, O. Callot, A. Cordier, M. Davier, L. Duflot, J.-F. Grivaz, Ph. Heusse, A. Höcker, M. Jacquet, D.W. Kim, ${ }^{19}$ F. Le Diberder, J. Lefrançois, A.-M. Lutz, I. Nikolic, H.J. Park, ${ }^{19}$ I.C. Park, ${ }^{19}$ M.-H. Schune, S. Simion, J.-J. Veillet, I. Videau, D. Zerwas

Laboratoire de l'Accélérateur Linéaire, Université de Paris-Sud, IN ${ }^{2} P^{3}$-CNRS, 91405 Orsay Cedex, France

P. Azzurri, G. Bagliesi, G. Batignani, S. Bettarini, C. Bozzi, G. Calderini, M. Carpinelli, M.A. Ciocci, V. Ciulli, R. Dell'Orso, R. Fantechi, I. Ferrante, A. Giassi, A. Gregorio, F. Ligabue, A. Lusiani, P.S. Marrocchesi, A. Messineo, G. Rizzo, G. Sanguinetti, A. Sciabà, P. Spagnolo, J. Steinberger, R. Tenchini, G. Tonelli, ${ }^{26}$ C. Vannini, P.G. Verdini, J. Walsh

Dipartimento di Fisica dell'Università, INFN Sezione di Pisa, e Scuola Normale Superiore, 56010 Pisa, Italy

G.A. Blair, L.M. Bryant, F. Cerutti, J.T. Chambers, Y. Gao, M.G. Green, T. Medcalf, P. Perrodo, J.A. Strong, J.H. von Wimmersperg-Toeller Department of Physics, Royal Holloway \& Bedford New College, University of London, Surrey TW20 OEX, United Kingdom ${ }^{10}$

D.R. Botterill, R.W. Clifft, T.R. Edgecock, S. Haywood, P. Maley, P.R. Norton, J.C. Thompson, A.E. Wright

Particle Physics Dept., Rutherford Appleton Laboratory, Chilton, Didcot, Oxon OX11 OQX, United Kingdom ${ }^{10}$

B. Bloch-Devaux, P. Colas, S. Emery, W. Kozanecki, E. Lançon, M.C. Lemaire, E. Locci, B. Marx, P. Perez, J. Rander, J.-F. Renardy, A. Roussarie, J.-P. Schuller, J. Schwindling, A. Trabelsi, B. Vallage CEA, DAPNIA/Service de Physique des Particules, CE-Saclay, 91191 Gif-sur-Yvette Cedex, France ${ }^{17}$

S.N. Black, J.H. Dann, R.P. Johnson, H.Y. Kim, A.M. Litke, M.A. McNeil, G. Taylor

Institute for Particle Physics, University of California at Santa Cruz, Santa Cruz, CA 95064, USA ${ }^{22}$ 
C.N. Booth, R. Boswell, C.A.J. Brew, S. Cartwright, F. Combley, A. Koksal, M. Letho, W.M. Newton, J. Reeve, L.F. Thompson

Department of Physics, University of Sheffield, Sheffield S3 7RH, United Kingdom ${ }^{10}$

A. Böhrer, S. Brandt, V. Büscher, G. Cowan, C. Grupen, G. Lutters, P. Saraiva, L. Smolik, F. Stephan, Fachbereich Physik, Universität Siegen, 57068 Siegen, Fed. Rep. of Germany ${ }^{16}$

M. Apollonio, L. Bosisio, R. Della Marina, G. Giannini, B. Gobbo, G. Musolino, Dipartimento di Fisica, Università di Trieste e INFN Sezione di Trieste, 34127 Trieste, Italy

J. Putz, J. Rothberg, S. Wasserbaech, R.W. Williams

Experimental Elementary Particle Physics, University of Washington, WA 98195 Seattle, U.S.A.

S.R. Armstrong, L. Bellantoni, ${ }^{23}$ P. Elmer, Z. Feng, ${ }^{28}$ D.P.S. Ferguson, Y.S. Gao, ${ }^{29}$ S. González, J. Grahl, T.C. Greening, J.L. Harton, ${ }^{27}$ O.J. Hayes, H. Hu, P.A. McNamara III, J.M. Nachtman, W. Orejudos, Y.B. Pan, Y. Saadi, M. Schmitt, I.J. Scott, V. Sharma, ${ }^{25}$ A.M. Walsh, ${ }^{30}$ Sau Lan Wu, X. Wu, J.M. Yamartino, M. Zheng, G. Zobernig

Department of Physics, University of Wisconsin, Madison, WI 53706, USA ${ }^{11}$

\footnotetext{
${ }^{1}$ Now at DESY, Hamburg, Germany.

${ }^{2}$ Supported by Dirección General de Investigación Científica y Técnica, Spain.

${ }^{3}$ Now at Dipartimento di Fisica, Università di Lecce, 73100 Lecce, Italy.

${ }^{4}$ Also Istituto di Fisica Generale, Università di Torino, Torino, Italy.

${ }^{5}$ Also Istituto di Cosmo-Geofisica del C.N.R., Torino, Italy.

${ }^{6}$ Supported by the Commission of the European Communities, contract ERBCHBICT941234.

${ }^{7}$ Supported by CICYT, Spain.

${ }^{8}$ Supported by the National Science Foundation of China.

${ }^{9}$ Supported by the Danish Natural Science Research Council.

${ }^{10}$ Supported by the UK Particle Physics and Astronomy Research Council.

${ }^{11}$ Supported by the US Department of Energy, grant DE-FG0295-ER40896.

${ }^{12}$ Also at Supercomputations Research Institute, Florida State University, Tallahassee, U.S.A.

${ }^{13}$ Supported by the US Department of Energy, contract DE-FG05-92ER40742.

${ }^{14}$ Supported by the US Department of Energy, contract DE-FC05-85ER250000.

${ }^{15}$ Permanent address: Universitat de Barcelona, 08208 Barcelona, Spain.

${ }^{16}$ Supported by the Bundesministerium für Forschung und Technologie, Fed. Rep. of Germany.

${ }^{17}$ Supported by the Direction des Sciences de la Matière, C.E.A.

${ }^{18}$ Supported by Fonds zur Förderung der wissenschaftlichen Forschung, Austria.

${ }^{19}$ Permanent address: Kangnung National University, Kangnung, Korea.

${ }^{20}$ Now at CERN, 1211 Geneva 23, Switzerland.

${ }^{21}$ Also at CERN, 1211 Geneva 23, Switzerland.

${ }^{22}$ Supported by the US Department of Energy, grant DE-FG03-92ER40689.

${ }^{23}$ Now at Fermi National Accelerator Laboratory, Batavia, IL 60510, USA.

${ }^{24}$ Now at Max-Plank-Institüt für Kernphysik, Heidelberg, Germany.

${ }^{25}$ Now at University of California at San Diego, La Jolla, CA 92093, USA.

${ }^{26}$ Also at Istituto di Matematica e Fisica, Università di Sassari, Sassari, Italy.

${ }^{27}$ Now at Colorado State University, Fort Collins, CO 80523, USA.

${ }^{28}$ Now at The Johns Hopkins University, Baltimore, MD 21218, U.S.A.

${ }^{29}$ Now at Harvard University, Cambridge, MA 02138, U.S.A.

${ }^{30}$ Now at Rudgers University, Piscataway, NJ 08855-0849, U.S.A.
} 


\section{Introduction}

With the increase of the LEP energy up to $136 \mathrm{GeV}$ achieved during the autumn of 1995, a new domain was opened to the search for new particles in $\mathrm{e}^{+} \mathrm{e}^{-}$collisions. In particular, searches for supersymmetric particles [1] have been reported by ALEPH [2] and other LEP collaborations [3]. These studies were all performed under the assumption that R-parity is conserved. R-parity [4] is a multiplicative quantum number defined as $R_{P}=(-1)^{3 B+L+2 S}$, where $B$ and $L$ are the baryonic and leptonic numbers, and $S$ is the spin. It takes the value +1 for all standard particles and -1 for their supersymmetric partners. The main consequence of R-parity conservation is the stability of the lightest supersymmetric particle (LSP).

Searches for supersymmetric particles in Z decays were also performed by ALEPH [5] in the case of R-parity non-conservation. More specifically, it was assumed that R-parity is violated by a lepton number non-conserving coupling, i.e. via a $\lambda_{i j k} L_{i} L_{j} E_{k}^{c}$ term in the superpotential [6], where $i, j$ and $k$ are generation indices. The lightest neutralino, $\chi$, assumed to be the LSP, then decays into the following final states:

$$
\bar{\nu}_{i} \ell_{j}^{+} \ell_{k}^{-}, \bar{\nu}_{j} \ell_{i}^{+} \ell_{k}^{-}, \nu_{i} \ell_{j}^{-} \ell_{k}^{+}, \nu_{j} \ell_{i}^{-} \ell_{k}^{+} .
$$

In addition, the $\lambda_{i j k}$ coupling was assumed to be large enough to allow the $\chi$ lifetime to be neglected.

In this letter, the search for charginos and neutralinos with R-parity violation is extended, under the same assumptions, using the data samples of 2.8 and $2.9 \mathrm{pb}^{-1}$ collected by ALEPH at centre-of-mass energies of 130 and $136 \mathrm{GeV}$, respectively. In these samples, a total of 1644 hadronic events from $\mathrm{e}^{+} \mathrm{e}^{-}$annilhilation were selected [7], of which more than one half is due to radiative returns to the $Z$. A thorough description of the ALEPH detector can be found in Ref. [8], and an account of its performance as well as a description of the standard analysis algorithms in Ref. [9].

Charginos are pair produced in $\mathrm{e}^{+} \mathrm{e}^{-}$collisions with typical cross sections of several picobarns at $130-136 \mathrm{GeV}$. Each chargino decays into $\chi f \bar{f}^{\prime}$, where $f$ and $f^{\prime}$ belong to the same weak isodoublet $\left(e . g . e^{-} \bar{\nu}_{e}\right.$ or $\left.u \bar{d}\right)$. Both charginos may decay into $\chi \ell \nu$, or one may decay into $\chi$ thadrons instead, or both may decay into $\chi$ thadrons; in the following, these cases are referred to as "leptonic, mixed and hadronic modes", respectively. Each $\chi$ then decays into $\ell^{+} \ell^{-} \nu$ with $\mathrm{R}$-parity violation. The topologies arising from chargino pair production depend both on the chargino decay modes and on the $\chi$ mass. As shown in Fig. 1, four analyses were designed to select these topologies: one for the leptonic mode; one for both the mixed and hadronic modes in the case of a large $\chi$ mass; and, for low $\chi$ masses, one for the mixed mode and another one for the hadronic mode. The results obtained could be affected by competing direct $\mathrm{R}$-parity violating chargino decays, such as $\chi^{+} \rightarrow \ell_{i}^{+} \ell_{j}^{+} \ell_{k}^{-}$or $\chi^{+} \rightarrow \nu_{i} \bar{\nu}_{j} \ell_{k}^{+}$; this issue is addressed at the end of this letter.

Neutralino production in $\mathrm{e}^{+} \mathrm{e}^{-}$collisions also occurs with cross-sections reaching several picobarns at $130-136 \mathrm{GeV}$ in regions of the parameter space where the lightest neutralino is predominantly higgsino-like. (In the minimal supersymmetric extension of the standard model (MSSM), this corresponds to $M_{2} \gg|\mu|$, with standard notations.) 


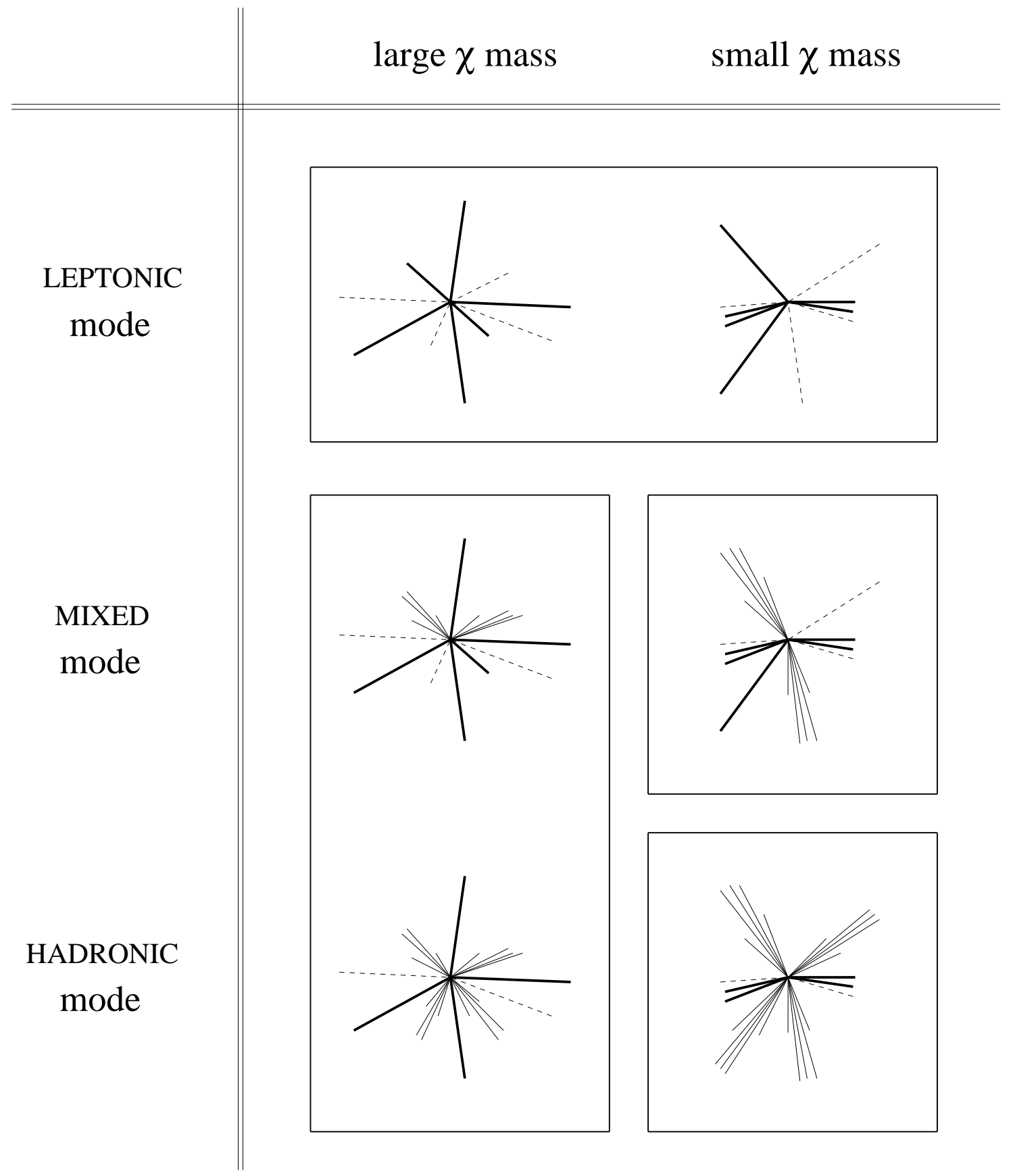

Figure 1: Chargino signal topologies as a function of the $\chi$ mass and of the chargino decay modes. Leptons are indicated by thick lines and neutrinos by dashed lines. The frames correspond to the four analyses performed to select these six topologies. 
In contrast to the case of $\mathrm{R}$-parity conservation, the $\mathrm{R}$-parity violating $\chi \rightarrow \ell^{+} \ell^{-} \nu$ decay renders visible the final state resulting from $\chi$ pair production. The topology which is to be selected consists of four leptons with some missing energy.

In the following, all efficiencies are given for the pair production of $65 \mathrm{GeV} / c^{2}$ mass charginos or neutralinos. Two extreme choices can be made for the $(i, j, k)$ triplet: $(1,2,2)$, in which case the $\chi$ decays into $\mu \mu \nu$ or $e \mu \nu$; or $(1,3,3)$, in which case the $\chi$ decays into $\tau \tau \nu$ or $e \tau \nu$. These configurations lead to the largest and smallest selection efficiencies. Conservatively, all results are given in the worst case, i.e. for a $\lambda_{133}$ coupling.

To design the selection criteria and to evaluate their efficiencies, the Monte Carlo program used to generate the chargino and neutralino signals was SUSYGEN [10], supplemented with a specific program [11] for the final state LSP decays. For all standard processes, Monte Carlo samples corresponding to an integrated luminosity at least three times as large as that of the data have been fully simulated. These standard backgrounds are the following: the annihilation processes $e^{+} e^{-} \rightarrow f \bar{f}$, the two photon scattering processes $\gamma \gamma \rightarrow f \bar{f}$, and the four fermion final states coming from the reactions $e^{+} e^{-} \rightarrow$ ZZ, Z $\gamma^{*}$ or $\gamma^{*} \gamma^{*}, e^{+} e^{-} \rightarrow$ Zee or $\gamma^{*} e e, e^{+} e^{-} \rightarrow \mathrm{W} e \nu$ and $e^{+} e^{-} \rightarrow$ WW. All these processes have been generated with the PYTHIA package [12], except for $\gamma \gamma$ scattering where the PHOT02 [13] program has been used.

\section{Event selections}

In the following, the same naming conventions as in Ref. [5] are used. In particular, the total, charged, photonic and neutral-hadronic energies refer to the energies carried by all reconstructed particles, by all charged particle tracks, by all photons and by all neutral hadrons (neutrons, $\mathrm{K}_{L}^{0} \mathrm{~s}$ ), respectively. The term "lepton" designates an electron or a muon, identified with the algorithms described in Ref. [9], but not a tau. The energy carried by the leptons is called "leptonic", and that carried by all the other particles "non-leptonic". Event hemispheres are defined by a plane perpendicular to the thrust axis. The acoplanarity is the angle between the projections of the hemisphere momenta onto a plane perpendicular to the beam axis. The transverse momentum designates the component of the total momentum transverse to the beam axis. Jets are reconstructed with the JADE algorithm [14], using charged and neutral particles. When the motivations for selection criteria are identical to those detailed in Ref. [5], they are not repeated here.

\subsection{Charginos in the leptonic mode}

At least five tracks, and not more than ten, are required. The visible mass must be in the range 20 to $90 \mathrm{GeV} / c^{2}$. The thrust must be smaller than 0.9 . The transverse momentum has to exceed $5 \mathrm{GeV} / c$. At this level, 19 events are selected in the data while 17.5 are expected (mostly from $\mathrm{e}^{+} \mathrm{e}^{-} \rightarrow \mathrm{q} \overline{\mathrm{q}}$ and $\gamma \gamma \rightarrow \mathrm{q} \overline{\mathrm{q}}$ ). Requiring at least two leptons, no events survive in the data. The small amount of expected background still remaining is eliminated by the requirements that the non-leptonic energy should not exceed $65 \mathrm{GeV}$ 
and that the neutral hadronic energy should be smaller than $10 \mathrm{GeV}$. For $\chi$ masses of 60 and $5 \mathrm{GeV} / c^{2}$ the selection efficiencies are 78 and $79 \%$, respectively.

\subsection{Charginos in the mixed and hadronic modes for large $\chi$ masses}

At least five tracks are required. The visible mass must be in the range 20 to $100 \mathrm{GeV} / \mathrm{c}^{2}$. The thrust must be smaller than 0.9. The angle between the thrust axis and the beam axis has to exceed $12^{\circ}$. The transverse momentum has to be larger than $7 \mathrm{GeV} / \mathrm{c}$. The acoplanarity is required to be smaller than $175^{\circ}$. There must be at least one lepton and the neutral hadronic energy must not exceed $8 \mathrm{GeV}$. At this level, five events are selected in the data while 5.9 are expected, mostly from $\mathrm{e}^{+} \mathrm{e}^{-} \rightarrow q \overline{\mathrm{q}}$. As can be seen in Fig. 2, requiring that the non-leptonic energy be smaller than $50 \mathrm{GeV}$ removes all data events. The remaining expected background is eliminated by the requirement that the longitudinal momentum be smaller than $25 \mathrm{GeV} / c$. The efficiencies are $60 \%$ in the mixed mode and $56 \%$ in the hadronic mode, for a $\chi$ mass of $60 \mathrm{GeV} / c^{2}$.

\subsection{Charginos in the mixed and hadronic modes for low $\chi$ masses}

At least five tracks are required, of which at least one must be a lepton. The transverse momentum has to be larger than $7 \mathrm{GeV} / c$. The other selection criteria are different for the mixed and hadronic modes.

For the mixed mode topology, the following requirements have to be fulfilled. The visible mass must be in the range 40 to $115 \mathrm{GeV} / \mathrm{c}^{2}$. The thrust must be smaller than 0.9. The acoplanarity must be smaller than $175^{\circ}$. The longitudinal momentum must be smaller than $30 \mathrm{GeV} / c$, the neutral hadronic energy smaller than $20 \mathrm{GeV}$ and the non-leptonic energy smaller than $90 \mathrm{GeV}$.

For the hadronic mode topology, the previous selection cannot be used because the hadronic energy as well as the visible mass are larger. Instead, it is required that the thrust be smaller than 0.85 , the visible mass between 70 and $125 \mathrm{GeV} / c^{2}$, the longitudinal momentum smaller than $15 \mathrm{GeV} / \mathrm{c}$ and the leptonic energy larger than $6 \mathrm{GeV}$.

After these cuts, nine events are found in the data by the mixed mode selection and ten by the hadronic one, to be compared to expectations of 6.0 and 7.7 events, mostly from $\mathrm{e}^{+} \mathrm{e}^{-} \rightarrow \mathrm{q} \overline{\mathrm{q}}$. In order to discriminate the signals against the remaining background, jets are reconstructed with a $y_{c u t}$ value of 0.0035 corresponding to an invariant mass of $8 \mathrm{GeV} / \mathrm{c}^{2}$. In Fig. 3, the number of reconstructed jets is shown as a function of the smallest of the jet charged multiplicities. For the mixed mode topology, at least four jets are required, and the smallest jet multiplicity must be 1,2 or 3 . For the hadronic mode topology, at least five jets are required, and the smallest jet multiplicity must not be zero. These cuts, designed to eliminate the remaining background, retain no events in the data. 

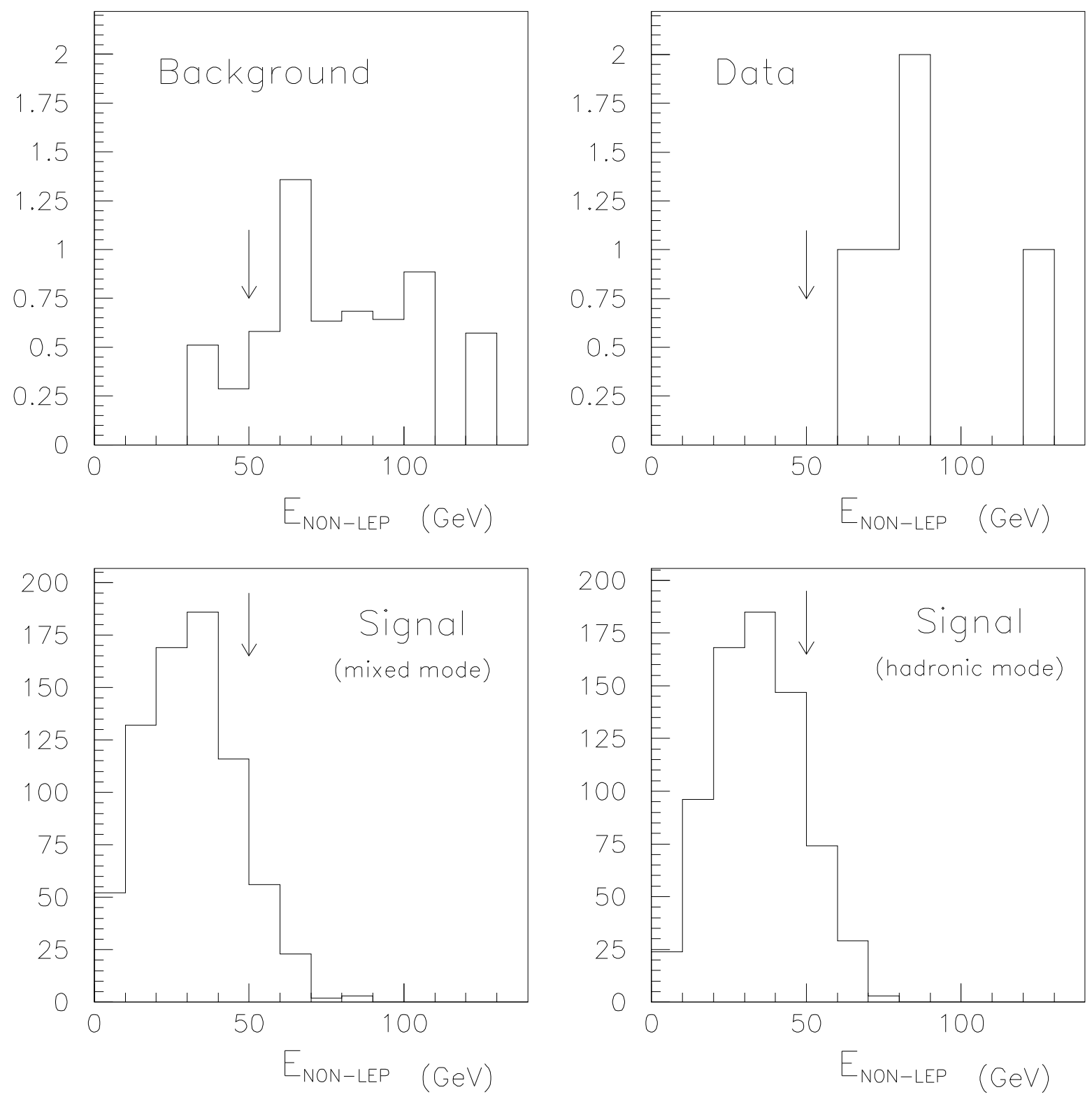

Figure 2: The non-leptonic energy $\mathrm{E}_{\mathrm{NON}-\mathrm{LEP}}$ for the background with an absolute normalization, for the data, and for a $65 \mathrm{GeV} / c^{2}$ mass chargino, with an arbitrary normalization, in the mixed and hadronic modes. The $\chi$ mass is $60 \mathrm{GeV} / c^{2}$, and the R-parity violating coupling is $\lambda_{133}$. The cut location is indicated by an arrow. 
The selection efficiencies are $64 \%$ and $46 \%$ in the mixed and hadronic modes, repectively, for a $\chi$ mass of $5 \mathrm{GeV} / \mathrm{c}^{2}$.

\subsection{Neutralino pairs}

To select $\chi \chi$ pairs, the following criteria are applied. The number of tracks must be at least four and not larger than eight, and the charged energy has to exceed $10 \mathrm{GeV}$. The visible mass must be in the range 15 to $95 \mathrm{GeV} / \mathrm{c}^{2}$. The transverse momentum is required to exceed $7 \mathrm{GeV} / c$. At this level, 39 events are selected in the data while 30.5 are expected. Jets are reconstructed with a $y_{\text {cut }}$ value of 0.00017 corresponding to the $\tau$ mass. The number of jets is shown in Fig. 4 as a function of the smallest of the jet charged multiplicities. The number of jets is required to be equal to four, and the smallest jet multiplicity must not be zero. To allow for hard final state radiation, events with five jets are also accepted if one and only one of these jets has zero multiplicity. Finally, a minimum of one lepton is required. These cuts eliminate the remaining background and select no events in the data. The selection efficiency is $61 \%$.

\section{Results}

Although each set of selection criteria was designed in view of a specific channel and for some specific $\chi$ mass range, most of these searches have a non-negligible efficiency for other channels and/or mass ranges. Therefore, for all the final states considered, the search efficiencies have been evaluated using the combination of all the selections described in Section 2. The improvement achieved this way can be seen in Table 1 . Here, the Rparity violating coupling is $\lambda_{133}$, leading to the lowest efficiencies. If a $\lambda_{122}$ coupling is considered instead, the combined efficiencies become $88 \%, 91 \%$ and $84 \%$ in the leptonic, mixed and hadronic modes, respectively, for a $60 \mathrm{GeV} / c^{2} \chi$ mass. Altogether, less than half an event is expected from the various background processes. No events were selected in the data.

In the MSSM, the masses and couplings of the charginos and neutralinos depend on three parameters: $m_{\tilde{\gamma}}$, the gaugino mass term associated with the photino field; $\mu$, a Higgs mixing supersymmetric mass term; and $\tan \beta$, the ratio of the vacuum expectation values of the two Higgs doublets. The production cross-sections and decay branching ratios also have some dependence on the various slepton masses. The results displayed in Fig. 5 were obtained assuming all sleptons to be substantially heavier than the weak gauge bosons.

The shaded region is the domain excluded by an analysis of the whole data sample collected by ALEPH at and around the $\mathrm{Z}$ peak from 1989 to 1995 . This result updates Fig. 7 of Ref. [5] which had been obtained ${ }^{1}$ with the 19891993 data. The domain excluded by the chargino searches at $130-136 \mathrm{GeV}$

\footnotetext{
${ }^{1}$ In Fig. 7 of Ref. [5], an error had been made in the determination of the black area showing the domain excluded by the search for $\mathrm{Z} \rightarrow \chi \chi$. The equivalent limit shown in Fig. 6 of Ref. [5] is however correct.
} 

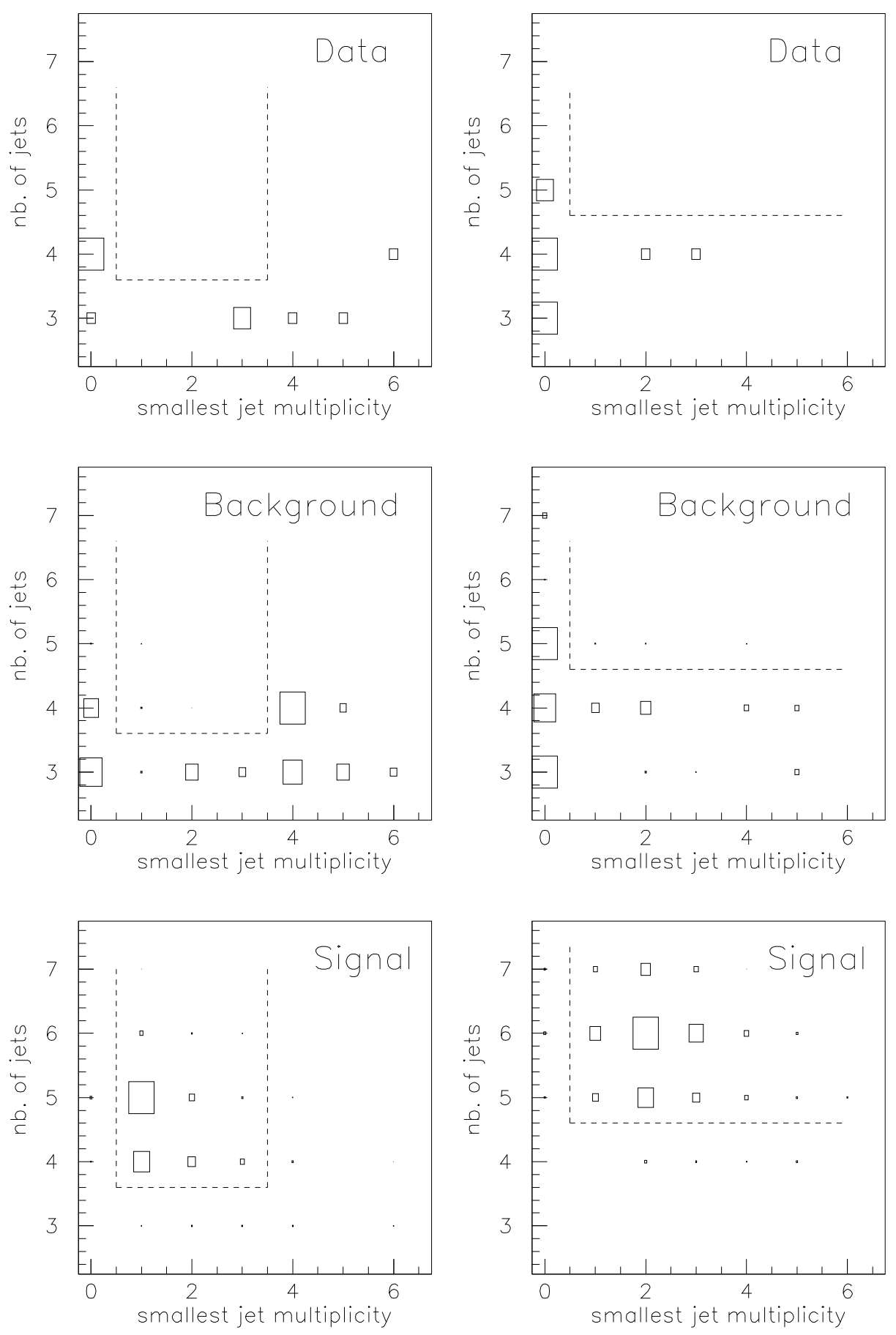

Figure 3: Number of jets vs. the smallest of the jet charged multiplicities for the mixed mode (left) and for the hadronic mode (right), for the data, the background, and a $65 \mathrm{GeV} / c^{2}$ mass chargino. The $\chi$ mass is $5 \mathrm{GeV} / c^{2}$, and the R-parity violating coupling is $\lambda_{133}$. The cuts are indicated by dashed lines. 

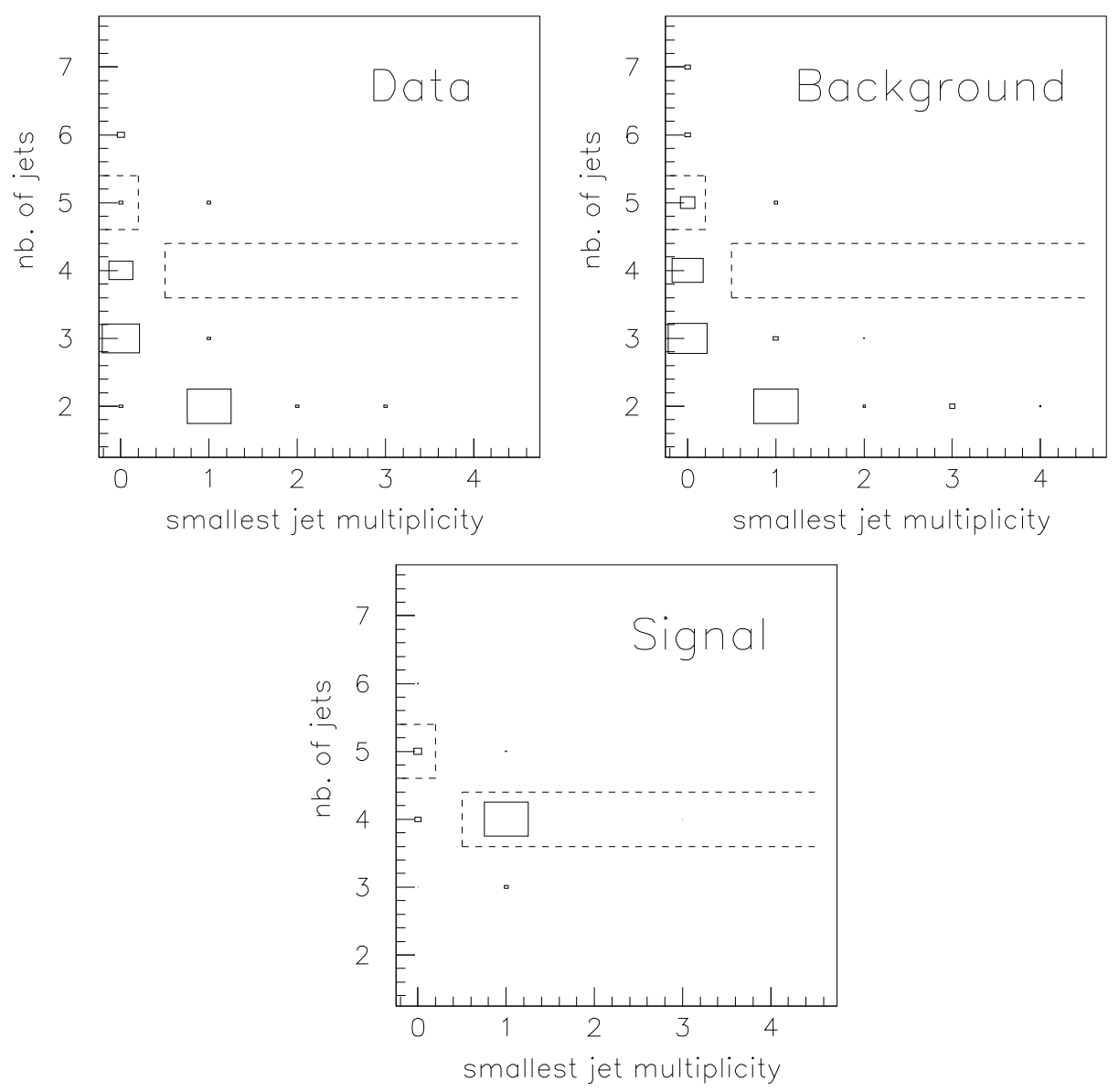

Figure 4: Number of jets vs. the smallest of the jet charged multiplicities for the data, the background, and the signal for $m_{\chi}=65 \mathrm{GeV} / c^{2}$. The R-parity violating coupling is $\lambda_{133}$. The cuts are indicated by dashed lines.

is limited by thick continuous lines, corresponding in practice to the kinematic limit of a $68 \mathrm{GeV} / \mathrm{c}^{2}$ chargino mass. The extension brought by the search for neutralinos is limited by thick dashed lines. Although dedicated searches for neutralino final states other than $\chi \chi$, such as $\chi \chi^{\prime}$, have not been performed, the combination of selections has been applied to these channels with substantial efficiency (e.g. $66 \%$ for $m_{\chi}=45 \mathrm{GeV} / c^{2}$ and $m_{\chi^{\prime}}=80 \mathrm{GeV} / c^{2}$ ).

In contrast to the case where R-parity is conserved, the extension of the domain excluded at LEP1 brought by the chargino and neutralino searches at $130-136 \mathrm{GeV}$ is very modest in the higgsino region $\left(m_{\tilde{\gamma}} / m_{Z}>0.8\right)$. This is because the luminosity accumulated at LEP1 has been sufficient to reach the kinematic limit in this region for $\mathrm{Z} \rightarrow \chi \chi$, a final state which is visible only when $\mathrm{R}$-parity is violated. On the other hand, the extension brought in the gaugino region $\left(m_{\tilde{\gamma}} / m_{Z}<0.4\right.$ and $\left.\mu / m_{Z}<-0.5\right)$ by the chargino searches at $130-136 \mathrm{GeV}$ is quite substantial for low values of $\tan \beta$. For values of $\tan \beta$ larger than 2 (not shown), the boundary of the excluded domain essentially coincides 

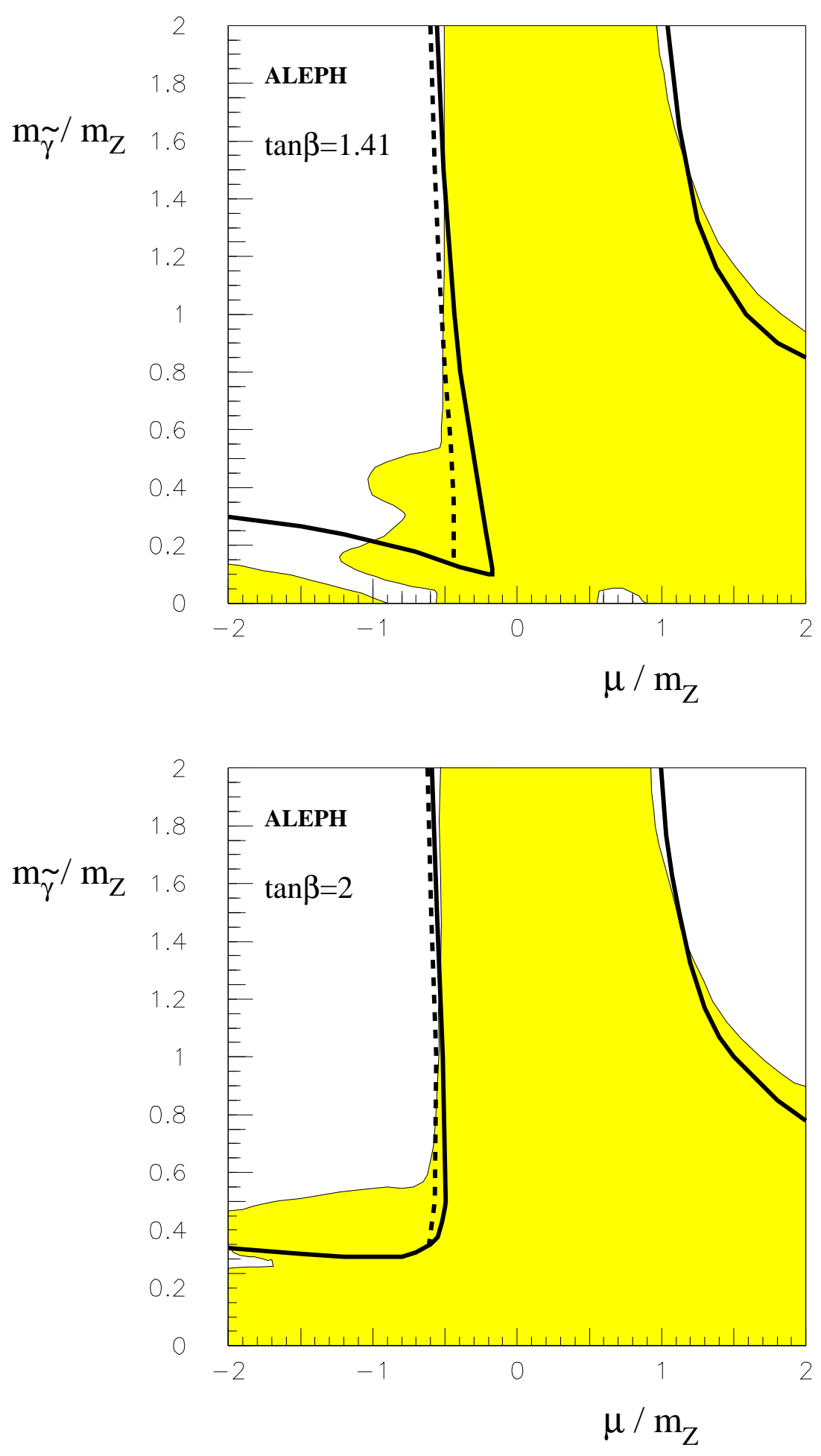

Figure 5: Domains in the MSSM $\left(m_{\tilde{\gamma}} / m_{Z}, \mu / m_{Z}\right)$ plane excluded at $95 \%$ C.L. at LEP1 (shaded), by the chargino search at $130-136 \mathrm{GeV}$ (heavy solid line) and by the neutralino search at $130-136 \mathrm{GeV}$ (dashed line), for two values of $\tan \beta$. 


\begin{tabular}{c||c||cc} 
Channel & $m_{\chi}$ & Specific & Combined \\
\hline \hline \multirow{2}{*}{ LEPTONIC } & 60 & 78 & 86 \\
& 5 & 79 & 87 \\
\hline \multirow{2}{*}{ MIXED } & 60 & 60 & 83 \\
& 5 & 64 & 71 \\
\hline \multirow{2}{*}{ HADRONIC } & 60 & 56 & 77 \\
& 5 & 46 & 50 \\
\hline$\chi \chi$ & 65 & 61 & 73
\end{tabular}

Table 1: Efficiency values, in percent, for the four final states considered and for various $\chi$ masses, for the specific and combined selections. For the leptonic, mixed, and hadronic mode selections, the chargino mass is $65 \mathrm{GeV} / \mathrm{c}^{2}$. The R-parity violating coupling is $\lambda_{133}$.

with the kinematic limit for $\mathrm{Z} \rightarrow \chi \chi$. For light sneutrinos, destructive interference affects the chargino pair production cross-section, reducing the chargino mass limit to $65 \mathrm{GeV} / \mathrm{c}^{2}$ in the worst case.

As mentioned in the introduction, these results could be invalidated if direct R-parity violating chargino decays had sizeable branching ratios [15]. The final states resulting from chargino pair production could then consist of six leptons, of four leptons and two neutrinos, or of two leptons and four neutrinos. While the two latter topologies have been analysed here and in Ref. [2], the six lepton final state has not been investigated. In the gaugino region however, direct R-parity violating decays contribute only at the percent level when the low energy constraints on R-parity violating couplings are imposed [16]. In the higgsino region, these branching ratios are somewhat larger although not dominant, but there the main constraint comes from neutralino production. Therefore, the results shown in Fig. 5 are unaffected.

\section{Conclusions}

In a data sample of $5.7 \mathrm{pb}^{-1}$ collected by the ALEPH detector at LEP at centre-of-mass energies of 130 and $136 \mathrm{GeV}$, searches for charginos and neutralinos have been performed under the assumption that R-parity is not conserved. It was assumed that the dominant R-parity violating coupling is of the $\lambda_{i j k} L_{i} L_{j} \bar{E}_{k}$ type, that the lightest neutralino is the LSP and that its lifetime can be neglected. No candidate events were found, which excludes charginos up to the kinematic limit of $68 \mathrm{GeV} / \mathrm{c}^{2}$ (for heavy sneutrinos) and restricts the allowed domain in the MSSM parameter space as shown in Fig. 5. 


\section{Acknowledgements}

We wish to thank and congratulate our colleagues from the accelerator divisions for having been so efficient in bringing up and operating LEP in this new energy regime. We are indebted to the engineers and technicians in all our institutions for their contribution to the excellent performance of ALEPH. Those of us from non-member countries thank CERN for its hospitality. 


\section{References}

[1] For a compilation of review articles, see: Supersymmetry and Supergravity, Ed. M. Jacob, North-Holland and World Scientific, 1986.

[2] ALEPH Coll., D. Buskulic et al., Phys. Lett. B 373 (1996) 246.

[3] L3 Coll., M. Acciarri et al., Search for Supersymmetric Particles at 130 GeV $<\sqrt{s}<$ $140 \mathrm{GeV}$ at LEP, CERN-PPE/96-29;

OPAL Coll., G. Alexander et al., Search for Chargino and Neutralino Production Using the OPAL Detector at $\sqrt{s}=130-136 \mathrm{GeV}$ at LEP, CERN-PPE/96-20.

[4] G. Farrar and P. Fayet, Phys. Lett. B 76 (1978) 575.

[5] ALEPH Coll., D. Buskulic et al., Phys. Lett. B 349 (1995) 238.

[6] L.J. Hall and M. Suzuki, Nucl. Phys. B 231 (1984) 419.

[7] ALEPH Coll., D. Buskulic et al., Measurement of hadron and lepton-pair production from $e^{+} e^{-}$annihilation at centre-of-mass energies of 130 and 136 GeV, CERN PPE/96-045, to appear in Physics Letters B.

[8] ALEPH Coll., D. Decamp et al., Nucl. Inst. and Methods A 294 (1990) 121.

[9] ALEPH Coll., D. Buskulic et al., Nucl. Inst. and Methods A 360 (1995) 481.

[10] S. Katsanevas and S. Melachroinos, SUSYGEN, in Physics at LEPD, CERN 1996-01, Vol. 2, Eds. G. Altarelli, T. Sjöstrand, and F. Zwirner.

[11] M. Jacquet, Recherche de particules supersymétriques avec violation de la R-parité, Thèse de Doctorat, Université Paris 6, LAL 95-89.

[12] T. Sjöstrand, Comp. Phys. Comm. 82 (1994) 74.

[13] ALEPH Coll., D. Buskulic et al., Phys. Lett. B 313 (1993) 509.

[14] JADE Coll., S. Bethke et al., Phys. Lett. B 213 (1988) 235.

[15] H. Dreiner, S. Lola and P. Morawitz, Chargino Pair Production at LEP2 with Broken R-Parity: 4-jet Final States, hep-ph/9606364.

[16] V. Barger, G.F. Giudice and T. Han, Phys. Rev. D 40 (1989) 2987. 